\title{
On the psychophysical discriminability of somatosensory nerve action potential patterns with irregular intervals'
}

\author{
WILLIAM R. UTTAL AND PAMELA SMITH \\ THE UNIVERSITY OF MICHIGAN
}

In this paper we consider the problem of whether or not a series of irregularities in the interpulse intervals in a train of nerve action potentials can be discriminated better than a single gap in a regular train. We also consider the corollary problem-does an increase in the number of impulses in a burst with irregular intervals lead to a decrease in the threshold for irregularity, thus indicating some integrative capacity of the relevant neural decoding mechanism. Answers to these questions are obtained by means of a psychophysical test and are affirmative in both cases. This suggests that the irregularities which have been observed in the intervals between nerve action potentials in animal preparations are true information-carrying codes within a restricted temporal region. Because of certain critical points in the functions relating this sensitivity to the basic frequency of the nerve impulses, a group of several temporal regions is postulated in which time is dealt with differently by the somatosensory system.

In recent years there has been a considerable amount of research reported in the neurophysiological literature concerning the detailed measurement of the variability of interpulse intervals in the train of nerve action potentials resulting from sensory stimulation (For an excellent review of the general problem, see Moore, Perkel, \& Segundo, 1966.) This work has generally been characterized by an experimental paradigm in which a natural stimulus impinging upon the usual transducer elicits a train of neural activity. This activity is detected by electrodes capable of transducing the activity of single cells. The temporal pattern of the nerve action potentials is then analyzed to show long-term statistical trends as well as microchanges in the timing. It has been shown, for example, that the variability of interpulse interval is, like the mean frequency, functionally related to the stimulus amplitude (Buller, Nicholls, \& Ström, 1953; Werner \& Mountcastle, 1963).Werner and Mountcastle have suggested on the basis of these results that the variability of interpulse interval itself may be an information-carrying code for sensory information; i.e., they suggest the existence of a hypothetical variability detector at some higher level of the nervous system.

This neurophysiological experimental paradigm, however, is not adequate to demonstrate the sufficiency of the hypothesis that interpulse interval is truly an information-carrying code for one or another stimulus dimension. Werner and Mountcastle carefully indicate their agreement with this notion by describing their observed results merely as a possible neural code. A complete proof of sufficiency of the hypothesis that interval variability is conveying information in a biologically significant way requires tests whose results show that the information encoded by the variability, as such, is not lost as some higher level of the nervous system. If the information is lost, the variation of interpulse interval can, at best, be considered a sign of some utility to the neurophysiologist but psychophysically insignificant. (See Utta1, 1967, for a detailed discussion of the significance of the terms signs and codes and some possible associated conceptual artifacts.) From one point of view, the usual neurophysiological experimental paradigm, as important and as powerful as it has been, also is capable of forcing our thinking toward the commission of a misconception about the coding process. Unless we are willing to concede, a priori, an extremely high degree of economy in the nervous system, such that most, if not all, observable neurophysiological patterns are actually used as codes in one way or another, we must consider the likelihood that many of the observed patterns of response are going to be irrelevant. They may be reflections of some underlying metabolic variable or effects of the complicated electrochemistry of cell membranes (Calvin \& Stevens, 1967) without being truly involved in those processes which ultimately will be identified with behavioral states.

Reports of tests in which measurements are made of the effectiveness of a given temporal pattern on some subsequent response, are very infrequent for several reasons. First, natural stimuli cannot appropriately be used since they produce patterns of nervous activity which are essentially uncontrolled in regard to interval variability. Thus, when a stimulus acts on a transducer, it is the transducer properties rather than the temporal properties of the stimulus which in large part determine the microtemporal patterns. Furthermore, there is no guarantee with natural stimuli applied to the normal transducers that the information is not being encoded redundantly by several different parameters of nervous activity.

For these reasons, it is necessary to force the desired nerve action potential pattern with electrical 
pulse stimuli-the only stimulus energy capable of defining a temporal pattern in neurons free of the above-mentioned difficulties.

The second difficulty limiting our knowledge of this important problem revolves around the fact that the distinction between a sign and a true code requires not only that information carried by some suggested coding dimension be transferred across a single synapse, but also that the pattern of information be maintained up to the level at which some neural activity actually becomes identifiable with some behavioral response. The great powers of the electrophysiological technique unfortunately do not include the ability to track a neural response through the several synaptic relays.

In this context, it becomes clear that the only way in which we can distinguish between a true informationcarrying code and a sign is a behavioral test. The specific question we ask: Is it possible for the organism to distinguish between two patterns which differ only in terms of the variability of the interpulse interval? The specific test we use: a test of psychophysical discriminability.

In the electrophysiological literature, there is but one report of which we are aware which observes differences in response as a function of a forced temporal pattern of nerve impulses. Segundo, Moore, Stensaas, and Bullock (1963) have observed differences in postsynaptic responsiveness (as measured by the amplitude of the postsynaptic potential) in aplysia. They did find that the temporal pattern of the presynaptic spike action potentials did produce changes in the amplitude of graded postsynaptic potentials and in the number of spike responses in the postsynaptic neurons independent of mean frequency. Earlier we (Uttal, 1960) had reported that triplets of spike action potentials in peripheral nerves were also differentially effective as measured with psychophysical tests of subjective amplitude. However, in that case the differential sensitivity could be completely accounted for in terms of the summed amplitude of the compound action potentials as modified by sequential refractory periods.

The purpose of the present study is to determine what are the limits and characteristics of psychophysical discriminability of nerve action potential patterns with irregular intervals. The nerve action potentials are patterns forced by direct electrical stimulation of peripheral somesthetic nerves by electrical pulses.

It should be noted that for all practical purposes it is generally not possible to carry out this type of psychophysical experiment on any other than the somesthetic system. The deeply buried acoustic and visual nerves are inaccessible to direct electrical stimuli unless surgically exposed. In this context our experiments can be seen to speak directly to the problem of the discriminability of nerve action potential patterns while other very similarly designed experiments such as the elegant work of Pollack (1967), and the studies of Cardozo, Ritsma, Domburg, and Neelen (1966), in audition describe the information processing capabilities of the entire system, pooling the transduction, transmission, and decoding processes into a single information measure. Our experiments specifically concern the transmission codes in peripheral nerves. The nerve action potential patterns resulting from peripheral electrical stimulation are designated only at the level of the first neuron in the ascending somatosensory pathway. Beyond that, we do not know whether the specific pattern we have created is maintained. It is, however, not germane; for we are more concerned with the maintenance of the information contained in the variability than the variability itself. If the pattern is discriminable with a psychophysical test, then we do know that the information is maintained regardless of what subsequent encodings it may have been subjected to and that the variability at the level of the first order neuron is a true information-carrying code.

We should also point out in this introduction that the experimental procedure used in this present experiment is also beset by certain limitations. First, although we do use electrical pulses to drive the nerve action potential pattern, the evoked responses are not trains of spikes in a single nerve fiber but the simultaneous responses of large groups of fibers. The complications that this compound response might introduce are not known but must be considered as a possible source of artifact. The work of Casby, Siminoff, and Houseknecht (1963) suggests that it may not be too significant, however. Second, the behavioral response which we use is a psychophysical judgment made immediately after the stimulus presentation. Ours is, therefore, an experimental situation which falls under the general rubric of sensory studies. It is not at all clear, however, that other longer term effects of nerve action potential pattern might be manifested but not observable by our methods. One could criticize our work on the basis of some long-term memory effect which we cannot observe but which does differentially affect behavior.

In spite of these limitations this experimental approach does offer an additional and, we believe, powerful means of evaluating the significance of various temporal patterns as possible neural codes which is not possible in the conventional neurophysiological or psychophysical experiment.

\section{Subjects}

\section{METHOD}

Six undergraduate student Ss, five male and one female, participated in this experiment. All were paid hourly wages and had either participated in similar experiments earlier or were pretrained on a related task before formal data collection began. 
The data of only five Ss is used in some of the experiments because of attendance problems not related to experimental procedures.

\section{Apparatus}

We have previously (Uttal \& Krissoff, 1966) described the nature of the electrical pulse stimuli used in this experiment. The individual pulses were constant current regulated and lasted for $.5 \mathrm{msec}$. Throughout all of the experiments reported in this present paper the amplitude of the pulses was kept constant at $4 \mathrm{~mA}$. The particular pattern required for each experimental trial was generated under the control of a small digital computer which automatically controlled all experimental operations. Stimulus patterns were timed, responses acquired, and statistical evaluation of the data compiled on line by the computer. Final shaping of the pulses was accomplished by a series of pulse formers external to the computer. The electrical pulse stimuli were conveyed to the $\mathrm{S}$ through two test tubes containing body normal saline solutions. The $\mathrm{S}$ inserted the index and middle fingers of his left hand into the test tubes up to the first joint. He responded by pushing a switch with his right hand to one of two momentary contact positions. The $S$ was seated in a small ( $3 \times 3 \mathrm{ft}$ ) acoustical cubicle in subdued lighting.

\section{Procedure}

The general purpose of this experiment was to determine how well the human somatosensory system is able to deal with irregularities in the intervals of a train of nerve impulses. Since it is well established (see for example Uttal, 1960) that electrical pulse stimuli produce compound nerve responses in peripheral somatosensory nerves on a one-for-one basis, we can assume that proper timing of the stimulus train forces the nerve action potential pattern to follow exactly the same temporal pattern. Three experiments were carried out in this initial attempt to explore the parameters of human discriminability of nerve action potential patterns with irregular intervals.

\section{Experiment 1}

In the first experiment the $\mathrm{S}$ was required to select which one of two sequential bursts of 10 electrical pulses was irregular or rough. The two stimulus bursts separated by $1-3 / 4$ sec were generated by the computer which also randomly selected whether the first or second of the two bursts actually contained the irregular intervals. The nature of the irregularity in this experiment was that an otherwise constant interpulse interval (termed the basic interpulse interval) was "jittered" in a quasi-random fashion by adding to each interval some proportion of a correction factor or perturbation index called $R$. For each proportion of an $\mathrm{R}$ that was added to one of the intervals in a train, an equivalent proportion was subtracted from some other interval so that the total length of the train when jittered remained the same as that of the unjittered reference train.

The only exception to this occurred when the subtraction of some proportional part of $\mathrm{R}$ would result in an interval less than 8 msec. We know that an interval this short is well below the temporal acuity threshold. Subjective magnitude increases could therefore occur in this time region which could bias the results of our experiment. By limiting the minimum interpulse interval to a minimum value of $8 \mathrm{msec}$, we do unfortunately occasionally introduce some differences into the durations of the two stimulus bursts. Typically, however, the thresholds for irregularity were higher for the trials in which this duration difference existed and it therefore appears that for these trials other effects swamped out any cues the $S$ might have gotten from duration differences.

The proportions of the basic perturbation $R$ which were used included: $+R,+3 / 4 R,+1 / 2 R,+1 / 4 R$, $0 \mathrm{R},-1 / 4 \mathrm{R},-1 / 2 \mathrm{R},-3 / 4 \mathrm{R}$, and $-\mathrm{R}$. These nine perturbation values were added to the basic interpulse interval in random order to form a table of jittered intervals. The table was then used to define the characteristics of the jittered burst emitted by the computer.

The intervals of the jittered burst can, therefore, seem to be randomly selected in a constrained fashion such that each interval occurs only once. When $R$ is very small, the degree of irregularity is very small since even the largest perturbations added to the basis interpulse results in an interval only slightly different than the unperturbed one. When $R$ is large, then the difference between the largest and smallest interval can be considerable. Figure 1 is a drawing
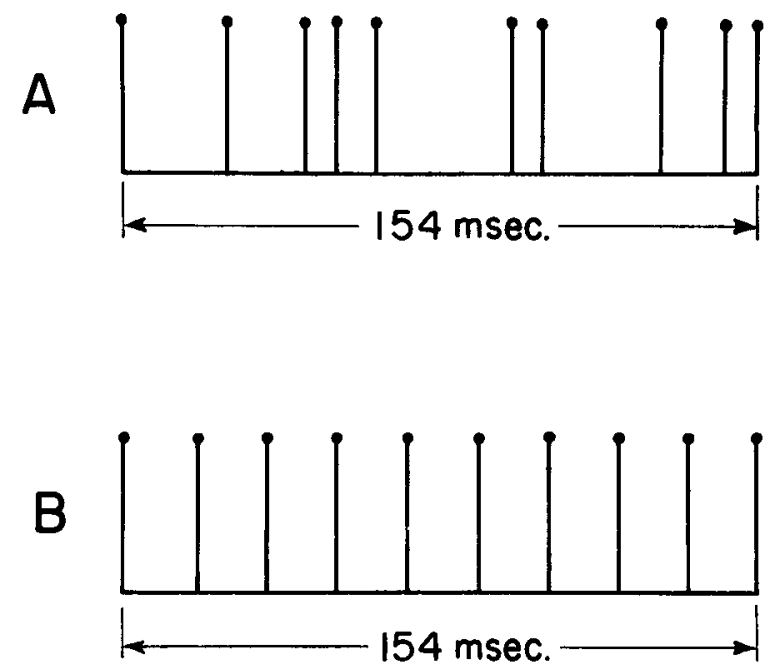

Fig. 1. A plot of two different stimulus bursts. $A$ is an irregular burst with jittered interpulse intervals. $B$ is a regular burst with a constant interval between serial pulses. These figures have been redrawn from oscillographic photographs. 
of a standard stimulus burst with equal interpulse intervals and a jittered stimulus burst showing the type of irregularity generated by these rules.

As mentioned previously, the independent variable in this experiment is the basic interpulse interval of the unjittered burst. The values of the basic interpulse intervals used in this experiment were 10, 12, 14, 16, 18, 20, 25, 30, and $40 \mathrm{msec}$. The dependent variable was the mean threshold size of $R$ for a just noticeable irregularity. An initial value for $R$ of $3 \mathrm{msec}$ was chosen at the beginning of each daily session with each $S$, but in subsequent blocks during that session $R$ was automatically set to the mean value of $\mathrm{R}$ for the previous block.

The psychophysical method used was a contingently designed two alternative forced choice procedure (as described by Green \& Swets, 1966) in which a correct answer ( $S$ selected the jittered burst) led to a reduction in the size of $R$ by $\Delta R$ and an incorrect response ( $S$ selected the regular burst) led to an increase in $R$ by $3 \Delta R$. No direct feedback of the correctness or incorrectness of his answer was given to the $S_{0} \Delta R$ itself was also set to an initial value of $1 \mathrm{msec}$ for each daily session but in each subsequent block was automatically set to a value equal to $1 / 10$ th of the range of $R$ on the previous block.

Within each block the contingent stimulus procedure precluded the prespecification of a detailed protocol; however, the computer was programmed to type out the value of the dependent variable and the S's answer upon each response. Within each trial the randomization procedure placed the longest interval $(\mathrm{IPI}+\mathrm{R})$ and the smallest interval (IPI-R) in unpredictable locations in sequential trials.

Each of the experimental conditions was presented once each day so that each daily session included nine blocks. The experiment was run for nine days with each day's blocks being presented in a different constrained random order. Each block consisted of 59 trials of which only the last 50 were used in the computations of the various statistical measures evaluated by the computer to avoid the effects of the initial hunting of transients from one condition to the next. Each point on the curve plotted in Fig. 2 therefore represents the mean of 2250 estimates by our group of Ss.

\section{Experiment 2}

One of the main possible sources of artifact in a design such as that used in Experiment 1 is that the $S$ might not be using all of the information which is presented to him by the jittered intervals. It may be that, for example, he is simply using the largest interval as a cue rather than the overall statistical structure of the stimulus burst. We have in the past reported a number of studies in which the parameters influencing the detection of a single gap in otherwise regular stimulus bursts were explored (see Uttal \&
Krissoff, 1966, 1967). To guard against the possibility of this sort of artifact, it was therefore necessary to compare the results for Experiment 1 with an equivalent set of data for the gap test. Our previous data, however, had been collected using an up-and-down method, of limits as our psychophysical procedure with a yes-no answer. It is well known that the data resulting from such a method will, in general, give higher thresholds than those obtained from a forced choice technique. Therefore, we replicated the study in which the effect of the size of the interpulse interval of a regular burst on the gap threshold was determined using a forced-choice technique. This experiment also used nine different interpulse intervals, but the interpulse intervals were all of the same size (except for the middle interval-the gap) in the burst of 10 pulses. Intervals of $10,12,14,16,18$, $20,25,30$, and $40 \mathrm{msec}$ were used. Nine daily sessions for each $S$ were run with each of the nine conditions being randomly presented (with the same constraint of no repetition as in Experiment 1) each day. Fifty responses by the $\mathrm{S}$ were used to determine the statistics of each block. The dependent variable in this case was simply the additional time which the $S$ on the average added to the basic interpulse interval for a threshold response. Each point on the curve plotted in Fig. 2, displaying the results of this experiment also, therefore, represents the average of 2250 estimates by our group of Ss.

\section{Experiment 3}

The third experiment considered a related problem. If the statistical sensitivity is observable, we should also expect that there would be some effect of the number of pulses in the train on the threshold for the detection of the irregularity in the jittered pulse train. This would be expected on the basis of the larger sample of interpulse intervals with which the $S$ could deal. In the third experiment, therefore, we varied the number of impulses in the jittered train but held the basic interpulse interval constant at 16 msec. Bursts containing $3,4,5,6,8,10,12,15$, and 20 pulses were used. Each block contained only one such number and nine blocks were presented in contrained random order each day for nine days. The same forced choice psychophysical procedure described in Experiment 1 was used in this experiment and the $S$ was instructed in this case also to respond by indicating which burst was irregular or rough. Each point on the curve drawn in Fig. 3 represents the average of 2700 estimates by our group of Ss.

\section{RESULTS}

The results of Experiment 1 and Experiment 2 are plotted on the same graph in Fig. 2 so that a direct comparison can be made between the two sets of data.

The results of Experiment 1 in which the Ss were 


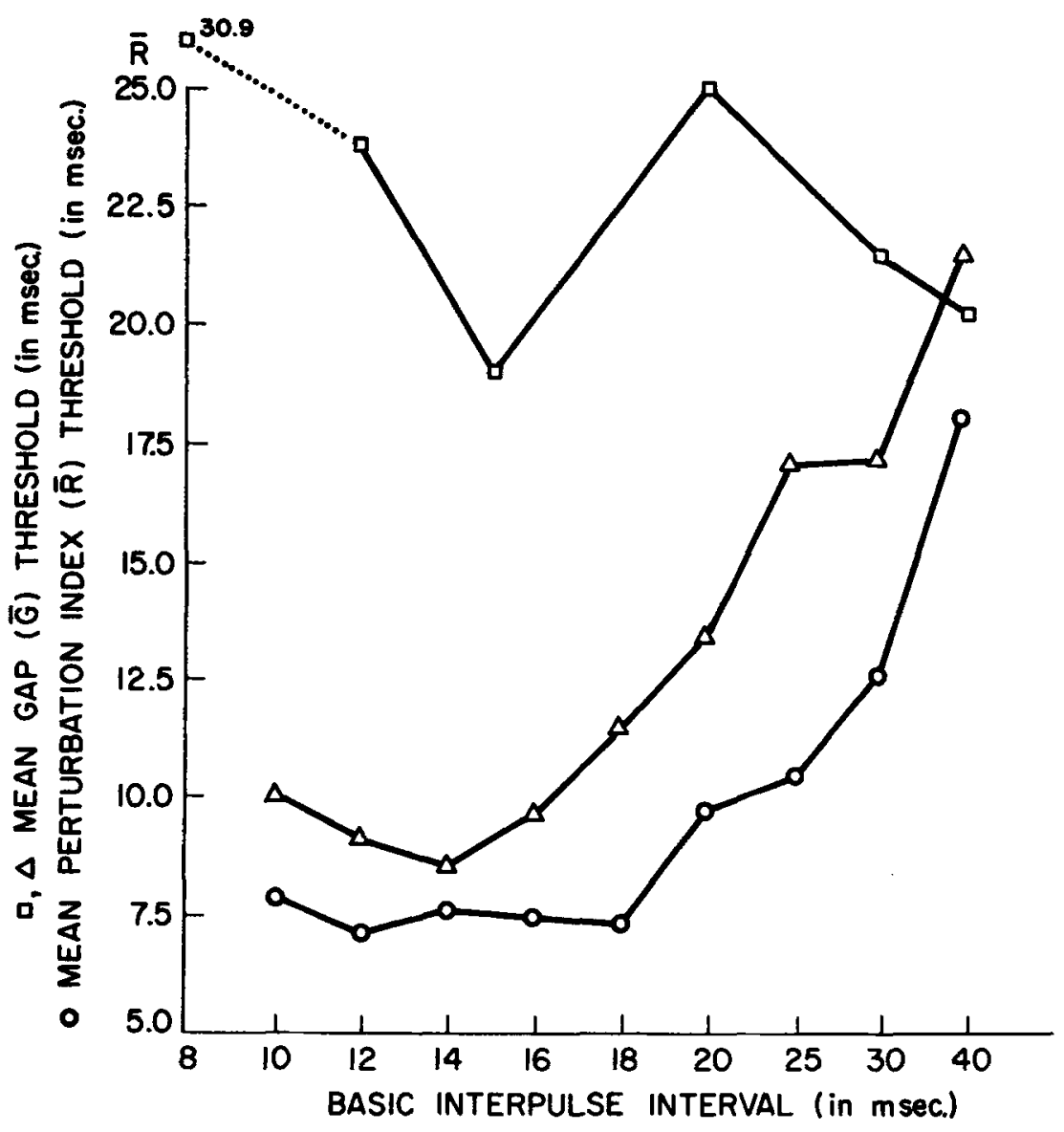

Fig. 2. The results of Experiments 1 and 2 and some older data for gap detection. The points plotted with circles are the results of the roughness detection experiment with a forced choice technique. The points plotted with triangles are the results of the gap detection experiment obtained with a forced choice technique. The points plotted with squares are the results of a gap detection experiment measured with a yes-no procedure. Data for five $\mathrm{Ss}$ in each case. Data for the gap thresholds are given in terms of additional time required above the basic interpulse interval for a just detectable gap.

asked to make a choice of which of the two bursts was "rough" are plotted with circles. The resulting functional relationship between the basic frequency and the just detectable amount of jitter appears to be made up of two segments. Below a basic interpulse interval $(20 \mathrm{msec})$ the $\mathrm{Ss}$ displayed a sensitivity to a constant amount of jitter independent of the basic interpulse interval. For intervals above $20 \mathrm{msec}$, however, the Ss required increases in the amount of jitter for detectability as the interpulse interval increased.

The data for Experiment 2 (the replication of our earlier experiment, in which the $S$ was required to detect a gap) are shown by the points plotted with triangles on Fig. 2. This curve also displays one effect obtained in our earlier experiment wherein it was shown that there was an elevation of the threshold at the very shortest intervals. The minimum detectable gap for this experiment was found at $14 \mathrm{msec}$ rather than at the $10 \mathrm{msec}$ basic interpulse interval.
However, comparison of this data with the results (points plotted with squares) of the earlier experiment which used the yes-no psychophysical procedure does show an important difference in the results. The yesno technique resulted in just detectable gap sizes which were essentially constant (except for the very shortest interval) over the entire range of the values of the basic interpulse interval used. There is general agreement between the methods for the largest values used. But, for the smaller values, there is a substantial difference between the two sets of data. The forcedchoice technique leads to substantially smaller thresholds in this region.

The results of Experiment 2, in which the number of pulses in the train was varied, are shown in Fig. 3. It can be seen from this data that the detectability of the irregularity does increase as the number of pulses in the burst of stimuli pulses increases. Apparently after a certain amount of information has been acquired by the $S$, additional information serves 
no further purpose and is redundant. The increase in effect is seen to persist out to a count of about eight pulses and then level off at about the value for the $16 \mathrm{msec}$ interval in Experiment 1.

\section{DISCUSSION}

The basic question which we ask in this study is whether or not Ss are able to discriminate between bursts of impulses with regular interpulse intervals and those with slightly irregular intervals. The corollary to this question is: Can Ss integrate, over time, a series of irregularities to give a more precise answer than that obtainable from a single interval of maximum divergence? The data answering these questions appears in both Fig. 2 and Fig. 3. In Fig. 2 we see that the threshold for an irregularly intervaled burst is lower than any value of the single gap test. The closest approach between the two curves is found at an interpulse interval of $14 \mathrm{msec}$. There is, however, an additional source of bias which suggests that the difference might be even larger under completely equitable conditions. The computer program generating the series of intervals in the irregular burst places the largest interval (basic interpulse interval $+\mathrm{R})$ at a random location in the burst. Thus, the largest interval could appear equally often at the middle of the burst or at either end. We have previously (Uttal \& Krissoff, 1966) shown that the posi- tion of the gap in the gap test paradigm significantly affected the threshold for its detection. The lowest threshold occurred when the gap was centrally placed in the train and thresholds were elevated when the gap was displaced to one side or the other of center. Assuming that the largest gap plays an especially important role in the detection of the statistical irregularity, then the random positioning of that particular interval throughout the pulse burst would be expected to lead to a general overestimate of the size of the threshold. Thus the difference between the two curves may be somewhat underestimated in our data.

Experiment 3 (in which the number of pulses was varied) also shows a substantial decrease in the threshold for the detection of the irregularity as the number of pulses increases up to the limit of about eight pulses. This suggests that the Ss are able to make use of the additional information being made available to them in a statistical fashion but only up to a certain point. This should be contrasted against the negligible effect of the number of pulses on the gap detection test reported in our earlier paper (Uttal \& Krissoff, 1966). This newer result, however, is ambiguous in that it may define either an integration requiring a certain number of pulses or an integration period. We are currently attempting to select which one of these two possible hypotheses

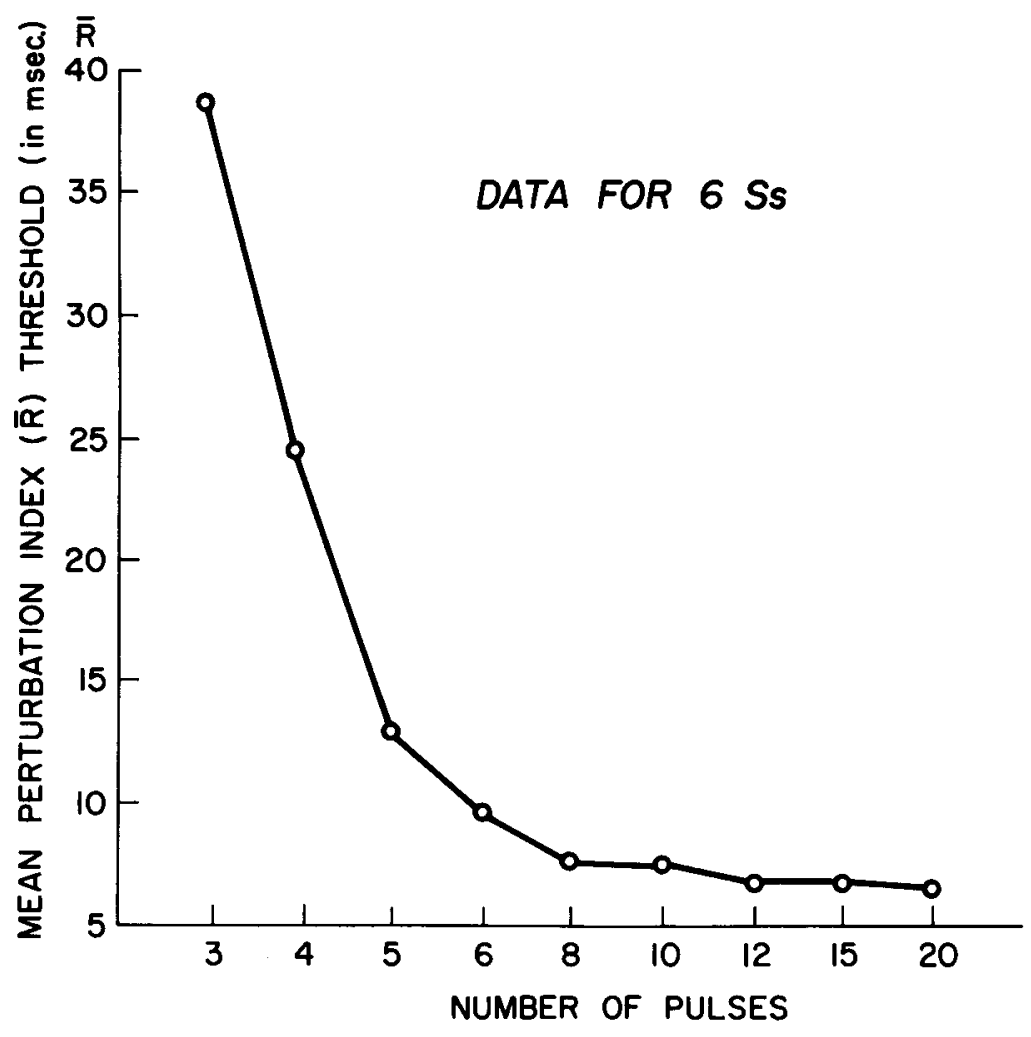

Fig. 3. The effect of number of pulses on the detectability of roughness. 
is the correct one by means of a study parametric in both $n$, the number of pulses, and $I$, the basic interpulse interval.

Another piece of evidence supporting the notion that the $S$ is able to make use of the statistical information in a way quite different from the way he might if he were only responding to the single largest gap is that the threshold for irregularity (as indicated by the size of $R$ ) is independent of the basic interpulse interval up to $20 \mathrm{msec}$. This is in contrast to the data for the gap test which shows a more complicated functional relationship between the size of the gap and the basic interpulse interval.

Let us now concern ourselves with the problem of the discrepancy between the data for the gap detection experiment as measured with a yes-no test and as measured with a forced-choice technique. Psychophysical testing with different methods usually results in somewhat different results. The $S$ is, in effect, being trained to make his judgments on the basis of different criterion levels in each case. There is no a priori validity of one or the other set of results. In the current experiment, however, we are primarily interested in defining the information transmission limits of the nervous system. This adds a special criterion for our choice of a specific psychophysical technique which does make one more "valid" than another. That criterion is that any technique used which genuinely lowers the threshold is considered superior. A finer discrimination, as evidenced by lower threshold, demonstrates an ability to distinguish between two stimuli which had been classified as indistinguishable. As such it meaningfully alters our estimate of the information transmission capacity of the nervous system. If we had, on the other hand, been studying the nature of decision-making, the difference in psychophysical methods could not be ordered along a continuum of validity, but rather would simply represent two different conditions of an independent variable which itself is of some intrinsic consequence. We now submit the results of Experiment 2 as a more up-to-date estimate of the additional time which must be added to the gap at each basic interpulse interval for a just detectable response. In the earlier paper (Uttal \& Krissoff, 1966) we had concluded that the additional time was a constant over a wide range. We no longer can accept that notion in the context of this newer data.

Another very significant feature of the curve relating the basic interpulse interval and the size of $R$ required for detection of roughness is the existence of two separate segments. This feature is very similar to one of the results reported by Mountcastle, Talbot, Darian-Smith, \& Kornhuber (1967) for the detection of vibratory stimuli. They found that the curve was also made up of two segments with the point of demarcation between the two occurring at about the same place on the frequency scale, as indicated on our chart. They concluded from their results that there were two different somatosensory systems operating in the detection of vibratory activity. The first was sensitive to low frequencies and was mediated by receptors located in the skin. The second was sensitive to higher frequencies and was mediated by receptors buried deep in the tissues of the hand. The differences in the receptors that were activated, they suggested, were directly dependent upon the frequency of the mechanical vibration. It is similarly possible that the two-segment curve obtained in this experiment also was a function of two different sets of nerve fibers. However, considering the nature of our stimulus, there is no reason to assume that the pulses affect different fibers as their frequency of occurrence is increased. It is more likely that since our stimuli activate large numbers of the neurons in the nerve trunk and even though almost all fibers are active, there is some segregation on the basis of the temporal processing capabilities of two groups of neurons. On the other hand, it is also possible that the two segments of the response curve do not represent two different groups of receptors or fibers but rather represent a dual characteristic curve for temporal processing in a uniform family of fibers.

As an alternative explanation of the two-segment curve reported by Mountcastle and co-workers and by our laboratory, we suggest the following hypothesis. Earlier (Uttal, 1960) we had shown that Ss are relatively insensitive to the temporal properties of a short burst of three stimulus pulses, all occurring within $10 \mathrm{msec}$, if one corrected for amplitude changes due to refractory effects. This may be the explanation for the increase in the threshold as the basic interpulse interval decreases below $14 \mathrm{msec}$ on the gap test and the slighter suggestion that the detectability of the jittered pulse train is also less sensitive at $10 \mathrm{msec}$.

On the basis of the present data we may speculate that there is a continuum of several regions of differing temporal processing capabilities as the frequency spectrum is scanned. Below $10 \mathrm{msec}(100 \mathrm{~Hz})$ there is a region of fusion in which there is generally a very low sensitivity to the temporal characteristics of the signal. In this time zone, the number of impulses is more important. Above $10 \mathrm{msec}$ and below $20 \mathrm{msec}$ there is a region in which there is an independent sensitivity to the second order statistics-the irregularity-of the interpulse intervals. Above $20 \mathrm{msec}$ one crosses into a region in which the mean interpulse interval is so large that the integrative powers of the nervous system are no longer capable of taking advantage of such higher order statistical data and judgments are made on the basis of mean interval or some independent measure of the size of local intervals. 


\section{References}

Buller, A. J., Nicholls, J. G., \& Ström, G. Spontaneous fluctuations of excitability in the muscle spindle of the frog. J. Physiol. (London), 1953, 122, 409-418.

Calvin, W. H., \& Stevens, C. F. Synaptic noise as a source of variability in the interval between action potentials. Science, $1967,155,842-844$.

Cardozo, B. L., Ritsma, R. J., Domburg, G., \& Neelen, J. J. M. Unipolar pulse trains with perturbed intervals; perceptibility of jitter. IPO Ann. Profr. Rep., No. 1 (Inst. Perception Res., Eindhoven, Holland), 1966, 17-27.

Casby, J. U., Siminoff, R., \& Houseknecht, T. R. An analogue cross-correlator to study naturally induced activity in intact nerve trunks. J. Neurophysiol., 1963, 26, 432-448.

Green, D. M., \& Swets, J. A. Signal detection theory and psychophysics. New York: John Wiley, 1966.

Moore, G. P., Perkel, D. H., \& Segundo, J. P. Statistical analysis and functional interpretation of neuronal spike data. Ann. Rev. Physiol., 1966, 28, 493-522.

Mountcastle, v. B., Talbot, w. H., Darian-Smith, I., \& Komhuber, H. H. Neural basis of the sense of flutter-vibration. Science, $1967,155,597-600$.

Pollack, I. Personal communication. 1967.

Segundo, J. P., Moore, G. P., Stensaas, L. J., \& Bullock, T. H. Sensitivity of neurones in aplysia to temporal pattern of arriving impulses. J. exp. Biol., 1963, 40, 643-667.

Uttal, W. R. The three pulse problem: A further comparison of neural and psychophysical responses in the somatosensory sy stem. J. comp. physiol. Psychol., 1960, 53, 42-46.

Uttal, W. R. Evoked brain potentials; signs or codes? Perspect. Med. Biol., 1967, in press.

Uttal, W. R., \& Krissoff, Madelon. Effect of stimulus pattern on temporal acuity in the somatosensory system. J. exp. Psychol., 1966, 71, 878-883.

Uttal, W. R., \& Krissoff, Madelon. On the refractoriness of somesthetic temporal acuity. Percept. \& Psychophys., 1967, 2, 115118.

Werner, G., \& Mountcastle, V. B. The variability of central neural activity in a sensory system. and its implications for the central reflection of sensory events. J. Neurophysiol., 1963, 26, 958977.

\section{Notes}

1. The research reported in this experiment was supported in part by NIH Grant MH08786-03 and in part by NSF Grants GB2000 and GB6093. We would also like to acknowledge the assistance of Mr. Peter Headly who wrote the control and analysis program for the computer.

(Accepted for publication April 19, 1967.) 